\section{El Movimiento de Salud de los Pueblos: salud para todos ya}

\author{
Miguel San Sebastián, ${ }^{1}$ \\ Anna-Karin Hurtig, ${ }^{1}$ \\ Jaime Breilh ${ }^{2}$ \\ y Arturo Quizhpe Peralta ${ }^{3,4}$
}

Palabras clave: formulación de políticas, política de salud, atención primaria de salud, Américas.

\footnotetext{
1 Escuela Internacional de Salud Pública de Umeå, Universidad de Umeå, Umeå, Suecia. La correspondencia debe dirigirse a Miguel San Sebastián, Umeå International School of Public Health, Epidemiology and Public Health Science, Public Health and Clinical Medicine, Umeå University, SE-901 85 Umeå, Sweden. Correo electrónico: miguel.sansebastian@epiph.umu.se

2 Centro de Estudios y Asesoría en Salud, Quito, Ecuador.

3 Facultad de Ciencias Médicas, Universidad de Cuenca, Ecuador.

4 Coordinación de la II Asamblea Mundial de Salud de los Pueblos, Cuenca, Ecuador.
}

Nos alejamos del año 2000 y el objetivo de salud para todos, reiterado en la Conferencia Internacional sobre Atención Primaria de Salud celebrada en 1978 en Alma Ata, antigua Unión Soviética, se encuentra todavía distante. De hecho pareciera que estamos dirigiéndonos en rumbo opuesto a dicho objetivo. Aunque se han mejorado ciertos indicadores de salud de la población en todo el mundo - como la esperanza de vida, la mortalidad infantil y la frecuencia de algunas enfermedades infecciosas y cardiovasculares-, existen importantes reveses (1). Las desigualdades entre los países y dentro de ellos aumentan y aún estamos lejos de controlar algunas enfermedades, como el sida, la malaria y la tuberculosis. La pérdida a gran escala del capital natural que constituye el medio ambiente está empezando a afectar a la capacidad de la biosfera para conservar una vida humana saludable a largo plazo. Con la promoción por las empresas transnacionales y las agencias internacionales - como el Banco Mundial y el Fondo Monetario Internacional- de un sistema económico basado en el mercado libre, se ha marginado el papel del Estado y ha aumentado el poder de esas grandes empresas, con lo que se cierran espacios para una conducción democrática de la gestión en salud. Muchos ven la globalización económica actual como una de las amenazas más graves a la salud de las poblaciones (2). En los últimos años, por ejemplo, se han promovido reformas en el sector de la salud orientadas al mercado, con la apertura de los servicios públicos a los inversores extranjeros y a los mercados mediante acuerdos comerciales internacionales. Este proceso indudablemente ejercerá una enorme presión en el espacio regulador social y medioambiental de los gobiernos nacionales y establecerá limitaciones a aquellas instituciones dedicadas a la salud pública y al bienestar social (3).

Junto a la globalización económica se observa también la globalización de las políticas internacionales de salud. En los últimos años, nuevos actores como el Banco Mundial, la Organización Mundial del Comercio, algunas multinacionales (por ejemplo, las de la industria farmacéutica) y nuevos filántropos (como la Fundación Gates) han entrado en el ruedo y han relegado a la Organización Mundial de la Salud (OMS) a un segundo plano (4). Todo ello ha contribuido a fomentar una mentalidad neoliberal en el campo de la salud, donde muy pocos y poderosos actores se encargan de establecer la agenda. Esto ha originado una manera de formular políticas 
de salud en línea con una ideología que favorece el crecimiento económico y la ayuda internacional como objetivos en sí mismos, en lugar de fomentar la redistribución de los recursos y el logro de la equidad (5). Además, hay indicios de que estas prescripciones políticas propagadas internacionalmente se basan más en estas ideologías que en análisis empíricos de lo que realmente funciona (6).

A partir de 1998 se observó una tendencia de cambio en las políticas de la OMS que coincidió con la elección de la nueva Directora General, cuando una serie de proyectos sobre temas económicos en torno a las políticas de salud, los servicios de salud y la salud pública culminaron en la publicación del Informe sobre macroeconomía y salud (7) y del Informe sobre la salud en el mundo 2000 (8). Quienes critican el primer informe han resaltado que concentrar la atención en la productividad económica y la mercantilización del derecho a la salud ha hecho que se pierda de vista que la salud es un derecho humano fundamental y ha fomentado una estrategia vertical de control de ciertas enfermedades, en lugar de fomentar el desarrollo de un sistema de salud integrado. Esto no es sino el reflejo de las políticas del Banco Mundial (9). En relación con el segundo informe, las críticas se centraron en el abandono por parte de la OMS de la meta de salud para todos, relegando la atención primaria de salud (APS) a una segunda generación de reformas de los sistemas sanitarios $(10,11)$. Aunque la OMS no ha negado oficialmente las políticas de APS (12), en muchos aspectos ha dejado a un lado algunos de los elementos esenciales de esa estrategia. Recientemente, el Informe sobre la salud en el mundo 2003 subrayó la necesidad de fortalecer los sistemas de salud basándose en los principios fundamentales de la APS formulados en Alma Ata (13). Si bien este enfoque es bienvenido, habrá que ver si este pronunciamiento es retórico o un compromiso real.

Con este contexto de fondo, se crea el Movimiento de Salud de los Pueblos (MSP) con el objetivo de restablecer el derecho a la salud integral y al desarrollo con equidad como principales prioridades en las políticas de salud en los niveles local, nacional e internacional, teniendo como meta alcanzar una APS integral como la proclamada en Alma Ata. La APS integral se articula con el pleno acceso a un programa integral de salud, de cobertura universal, con la garantía de acceso a los beneficios de acciones curativas en todos los niveles de complejidad y servicios preventivos en todos los ámbitos de la vida colectiva - como el trabajo- y la defensa de espacios de consumo saludables. Además, se debe garantizar el desarrollo de una gestión de salud democrática y acorde con el interés social, enriquecida interculturalmente con el aporte de todos, en armonía con un ambiente ecológico saludable y sin re- ducción a paquetes mínimos verticales focalizados en indicadores extremos (14).

\section{LA PRIMERA ASAMBLEA MUNDIAL DE SALUD DE LOS PUEBLOS}

A mediados de la década de 1980, diferentes movimientos de la sociedad civil iniciaron conversaciones sobre la necesidad de inyectar "realidad" a las políticas internacionales de salud. Varios años más tarde, en mayo de 1998, un grupo de miembros de organizaciones de la sociedad civil que estuvieron en Ginebra, Suiza, para la Asamblea Mundial de la Salud que celebra anualmente la OMS acordaron explorar la idea con mayor profundidad.

Estas conversaciones condujeron a la primera Asamblea Mundial de Salud de los Pueblos, celebrada en Bangladesh en diciembre de 2000, en la cual participaron más de 1500 delegados de 75 países. Muchos de ellos provenían de organizaciones de base $\mathrm{u}$ organizaciones que trabajaban de cerca con comunidades pobres y marginadas. En la Asamblea, cuyo tema fue "escuchar a los ignorados", se presentaron los puntos de vista de países de altos y de bajos ingresos y el análisis se combinó con experiencias vivas (15).

El objetivo del proceso que condujo a la primera Asamblea Mundial de Salud de los Pueblos y el de la propia Asamblea fue fortalecer los vínculos entre las personas y crear un movimiento internacional orientado a lograr que el objetivo de salud para todos volviera a ocupar un lugar principal dentro de la agenda de desarrollo internacional.

Uno de los logros importantes de la Asamblea fue la aprobación de la Declaración para la Salud de los Pueblos. La Declaración, que expresa las inquietudes colectivas y la visión de un mundo mejor y más saludable, constituye un llamado a la acción radical. Es una herramienta de reivindicación y un punto de encuentro para un movimiento mundial de salud conducente a formar nuevas redes y coaliciones. La Declaración se fundamenta en cinco principios (16):

- Lograr el más alto nivel de salud y bienestar es un derecho humano fundamental, sin importar el color de la piel, el origen étnico, la religión, el sexo, la edad, las habilidades, la orientación sexual o la clase social de la persona.

- Las políticas relacionadas con la salud deben basarse en la atención primaria universal e incluyente, principios concebidos en la Declaración de Alma Ata de 1978. Ahora más que nunca se requiere de un enfoque equitativo, participativo e intersectorial.

- Los gobiernos tienen la responsabilidad fundamental de garantizar el acceso universal a una 
atención sanitaria de buena calidad, a la educación y a otros servicios sociales, de acuerdo con las necesidades de la población y no según su capacidad de pago.

- La participación de la población y de las organizaciones populares es esencial para la formulación, puesta en marcha y evaluación de todas las políticas y programas sociales y sanitarios.

- La salud se ve determinada primordialmente por el ambiente político, económico, social y físico y debe ser asunto de gran prioridad al elaborar las políticas locales, nacionales e internacionales, junto con la equidad y el desarrollo sostenible.

\section{EL MOVIMIENTO DE SALUD DE LOS PUEBLOS}

Otro importante logro de la primera Asamblea Mundial de Salud de los Pueblos fue la creación del MSP, coalición de organizaciones de la sociedad civil, organizaciones no gubernamentales (ONG), activistas sociales, profesionales de la salud, académicos e investigadores que respaldan la Declaración para la Salud de los Pueblos.

Una de las principales tareas desempeñadas por el MSP ha consistido en identificar las dificultades comunes que enfrentan los diversos participantes y proponer acciones al respecto. La privatización de los servicios de salud, el impacto de los conflictos armados, la continua amenaza al medio ambiente y la introducción de acuerdos internacionales de libre comercio son algunas de las principales inquietudes identificadas en el nivel mundial.

El MSP ha desarrollado una estrategia para la comunicación que se basa en diversas vías, entre ellas una página en Internet (www.phmovement. org), una lista de correo electrónico para el intercambio y la discusión de ideas y documentos (phaexchange@kabissa.org) y noticieros y comunicados de prensa acerca de una amplia variedad de temas, especialmente de eventos.

Del 17 al 23 de julio de 2005, en Cuenca, Ecuador, se realizará la segunda Asamblea Mundial de Salud de los Pueblos, organizada por el MSP. Esta Asamblea será de nuevo la culminación de un proceso de reflexiones, discusiones y debates en los niveles local y nacional, y de intercambios de experiencias entre las comunidades y las redes internacionales. Esos debates se centran en los factores que ejercen un efecto determinante en la salud y el bienestar de las poblaciones. El diseño de una agenda para la construcción de una salud renovada como parte de ese otro mundo posible será una de las principales metas de la Asamblea.

El lema de esta Asamblea es "iLas voces de la tierra nos convocan!: construyamos un mundo sa- ludable". Entre sus ejes temáticos se encuentran la salud como derecho humano fundamental; la militarización y ocupación; la degradación ambiental; las pandemias emergentes y reemergentes; la equidad, la pobreza y la salud; la interculturalidad crítica; la violencia social y política; la salud en manos del pueblo; la salud en el trabajo; la medicina tradicional y bioenergética; el género; y la reforma del sector de la salud. Estos temas serán abordados con una visión amplia, integral y general mediante exposiciones analíticas y debates, así como relatos, estudios de casos, exposiciones fotográficas, dibujos, pinturas y artículos de artesanía.

Junto a las actividades anteriores se ha previsto realizar eventos especiales, tales como el Foro Global de la Niñez, el Festival de la Esperanza y la Alegremia, la ceremonia y la Declaración de los Pueblos Originarios, el Foro de la Juventud, la Marcha por la Paz, la Salud y la Dignidad de los Pueblos, y la presentación del Informe Alternativo de la Salud Mundial. Estos eventos tienen como propósito recoger las opiniones y fomentar el protagonismo de los grupos humanos más marginados $\mathrm{y}$ vulnerables (17).

\section{EL OBSERVATORIO MUNDIAL DE LA SALUD}

Durante la Asamblea Mundial de la Salud celebrada por la OMS en mayo de 2003, el MSP, junto con otras dos ONG (Global Equity Gauge Alliance y Medact) discutieron la posibilidad de que la sociedad civil elaborara un documento como alternativa al Informe sobre la salud en el mundo emitido por la OMS. Esta propuesta obedeció a la necesidad de ofrecer una visión alterna del estado de la salud mundial y plantear una perspectiva sobre la salud desde el punto de vista de los pueblos, independientemente de las estructuras oficiales de poder y de las agendas de las agencias de cooperación internacional. Este documento alterno debería servir para fortalecer la capacidad de los pueblos de luchar por la equidad y la vigencia de los derechos humanos y sociales y permitir el monitoreo de las políticas de las instituciones internacionales de salud, además de desafiar el discurso neoliberal sobre políticas de salud con una estrategia centrada en las personas y en la justicia social. La idea de un informe alterno culminó con la iniciativa denominada Observatorio Mundial de la Salud (Global Health Watch).

El primer Informe Alternativo de la Salud Mundial se presentará durante la II Segunda Asamblea Mundial de la Salud de los Pueblos ante delegaciones de todas las regiones del mundo. Para reforzar su impacto, este informe se presentará si- 
multáneamente en diversas ciudades. Los resultados y reflexiones del Informe se presentarán de forma estructurada en seis capítulos que se titulan: a) "Políticas y economía de la salud en el siglo XXI"; b) "El sector de la atención sanitaria"; c) "Más allá del sistema de salud"; d) "Los grupos excluidos y marginados"; e) "El monitoreo de las instituciones y de los flujos de recursos"; y f) "Síntesis y sumario de estrategias para la acción" (18).

En esta ocasión se ha decidido publicar complementariamente el Informe Alternativo de la Salud en América Latina, no solo en atención al hecho de que la Asamblea se realizará en un país latinoamericano, sino como reconocimiento a las valiosas contribuciones de esta subregión a la investigación alternativa en el campo de la salud. La comisión organizadora de la Asamblea ha delegado la responsabilidad de coordinar el diseño, la elaboración y la presentación del Informe Alternativo al Centro de Estudios y Asesoría en Salud (CEAS) de Quito, Ecuador, que celebrará 25 años de su producción científica.

El Informe Alternativo de la Salud en América Latina se ha concebido para ofrecer una visión clara, tanto de las consecuencias destructivas del modelo neoliberal de la salud como de los proyectos alternativos exitosos impulsados por algunos gobiernos y movimientos progresistas de la Región a pesar de las adversas condiciones impuestas por ese modelo social. El Informe estará estructurado en dos secciones. En la primera, denominada "La acumulación por despojo y la salud en países de América Latina", se expondrá el impacto del modelo neoliberal y de las correspondientes políticas de salud en algunos países latinoamericanos, mientras que en la segunda sección, titulada "Esa otra salud posible", se presentarán algunos resultados notables logrados por gobiernos democráticos de países y ciudades de la Región. Este informe se publicará en español e inglés.

\section{CONCLUSIÓN: SALUD PARA TODOS YA}

En septiembre del año 2000, representantes de 189 países se reunieron en la Cumbre del Milenio, convocada por la Organización de las Naciones Unidas, y adoptaron la declaración que dio base a la formulación de los Objetivos de Desarrollo del Milenio. Estos objetivos forman parte de una larga serie de iniciativas emprendidas por los gobiernos, el sistema de las Naciones Unidas y las instituciones internacionales de financiamiento para reducir la pobreza y el hambre, mejorar el estado de salud, reducir las desigualdades de género y la falta de educación, facilitar el acceso al agua potable y frenar la degradación ambiental en el mundo (19).
En las Américas, muchos indicadores de salud han mejorado en los últimos 20 años (20). Sin embargo, América Latina y el Caribe siguen teniendo las mayores desigualdades del mundo en cuanto a la distribución de los ingresos (21). Las desigualdades de salud entre los distintos países y áreas, y dentro de un mismo país, son enormes (22). No es seguro que América Latina y el Caribe puedan alcanzar los Objetivos de Desarrollo del Milenio para la fecha propuesta (23). Por ejemplo, solo $4,2 \%$ de los países latinoamericanos están en vías de reducir la mortalidad materna a los niveles propuestos en esos objetivos para el año 2015 (24).

Poder alcanzar los Objetivos de Desarrollo del Milenio dependerá principalmente de dos circunstancias: la disponibilidad de recursos económicos suficientes y la mejora de los sistemas nacionales de salud (25). Si bien la primera estrategia depende en parte del compromiso de los gobiernos de los países de bajos recursos, estos no podrán alcanzar esos objetivos a menos que los países industrializados establezcan compromisos a largo plazo para facilitarles nuevos recursos y acuerdos comerciales justos. Sin embargo, hasta el momento no se ha logrado cancelar la deuda externa, no se ha avanzado hacia la meta propuesta por las Naciones Unidas de destinar $0,7 \%$ del producto nacional bruto de los países de mayor desarrollo al impulso de las economías de los países más pobres, y siguen vigentes los actuales reglamentos comerciales, que pueden empeorarse aun más con los llamados "tratados de libre comercio" que favorecen solamente a los países industrializados $(26,27)$. La segunda estrategia, que consiste en mejorar los sistemas nacionales de salud, dependerá a su vez de cambios en la gestión del sector público, de que haya más investigación en el sector de la salud, así como del establecimiento de políticas e intervenciones que vayan más allá de dicho sector (25).

Estas estrategias, junto con las líneas específicas de acción, se recogen en la Declaración para la Salud de los Pueblos promovida por el MSP. La adopción de esta Declaración en los ámbitos nacional e internacional contribuirá, sin dudas, a alcanzar los Objetivos de Desarrollo del Milenio y a allanar el camino hacia una salud para todos, tanto en América Latina y el Caribe como en el resto del mundo.

\section{SYNOPSIS}

\section{The People's Health Movement: health for all now}

We are moving away from the year 2000, and the goal of "health for all" remains distant. Economic globalization, which many consider to be one of the most seri- 
ous threats to health, moves ahead, along with globalization of international health policies. A small number of powerful actors are in charge of setting the agenda, and they relegate to an inferior position the World Health Organization, which has moved away from the goal of "health for all." All of this has helped to promote a neoliberal mentality in the field of health. The People's Health Movement was created with the objective of reestablishing the right to comprehensive health and to development with equity as principal priorities of health policies at the local, national, and international levels. The People's Health Movement uses as a strategy the People's Charter for Health, which was approved at the first People's Health Assembly, which was held in Bangladesh in 2000. The Charter expresses collective concerns and the belief in a better and healthier world as a meeting point in order to promote a world health movement and a call for radical action. In July 2005 the People's Health Movement will hold the People's Health Assembly 2, in the city of Cuenca,
Ecuador. The slogan of that meeting will be "The voices of the earth are calling!-let's build a healthy world." Among the topics that will be examined at the meeting are: health as a fundamental human right; militarization and occupation; environmental degradation; emerging and reemerging pandemics; equity, poverty, and health; the importance of cultural diversity; social and political violence; health in the hands of the people; health at work; traditional medicine and bioenergetic medicine; gender; and health sector reform. The objective of the People's Health Movement is to help to reach the Millennium Development Goals and to smooth the path toward attaining health for all, in Latin America and the Caribbean as well as in the rest of the world.

Key words: policy making, health policy, primary health care, Americas.

\section{REFERENCIAS}

1. Sanders D. Twenty five years of primary health care: lessons learned and proposals for revitalization. En: People's Health Movement, ed. Health for all now, 2003. Hallado en: http://www. phmovement.org. Acceso el 25 de marzo de 2005.

2. McMichael A, Beaglehole R. The global context for public health. En: Beaglehole R, ed. Global public health: a new era. New York: Oxford University Press; 2003.

3. Labonte R. Liberalisation, health and the World Trade Organization. J Epidemiol Community Health. 2001;55:620-1.

4. Buse K, Drager N, Fustukian S, Lee K. Globalisation and health policy: trends and opportunities. En: Lee K, Buse K, Fustukian S, eds. Health policy in a globalizing world. Cambridge, England: Cambridge University Press; 2002.

5. Katz A. The Sachs Report: investing in health for economic development-or increase the size of the crumbs from the rich man's table? Part 1. Int J Health Services. 2004;34:751-73.

6. Brugha R, Zwi A. Global approaches to private sector provision: where is the evidence? En: Lee K, Buse K, Fustukian S, eds. Health policy in a globalizing world. Cambridge, England: Cambridge University Press; 2002

7. Commission on Macroeconomics and Health. Macroeconomics and health: investing in health for economic development. Geneva: World Health Organization; 2001.

8. World Health Organization. The World Health Report 2000. Geneva: WHO; 2000.
9. Waitzkin H. Report of the WHO Commission on Macroeconomics and Health: a summary and critique. Lancet. 2003; 361:523-6.

10. Almeida C, Braveman P, Gold MR, Szwarcwald CL, Mendes Ribeiro J, Miglionico A, et al. Methodological concerns and recommendations on policy consequences of the World Health Report 2000. Lancet. 2001;357:1692-7.

11. Cohen J. Worse than a crime; a mistake. Lancet. 2003;361:876-7.

12. Brundtland GH. Response from WHO Director General. Lancet. 2003;361:877.

13. World Health Organization. World Health Report 2003. Shaping the future. Geneva: WHO; 2003.

14. Werner D, Sanders D. Questioning the solution: the politics of primary health care and child survival. Palo Alto: HealthWrights; 1999.

15. Narayan R, Schuftan C. The Peoples's Health Movement: a people's campaign for "Health for all-Now". En: Harris RL, Seid M, eds. Globalization and health. Leiden, The Netherlands: Brill; 2004.

16. People's Health Movement. Declaración para la Salud de los Pueblos. Hallado en: http://www.phmovement.org/charter/ pch-spanish.html. Acceso el 28 de marzo de 2005.

17. Consejo Internacional de Salud de los Pueblos. Convocatoria a la Asamblea de Salud de los Pueblos 2. Hallado en: http://www.iphcglobal.org/index_es. htm. Acceso el 7 de abril de 2005.

18. Global Health Watch. Movilizando a la sociedad civil alrededor de un informe alternativo sobre la salud mundial. Hallado en: http://www.ghwatch.org/ index. html.es. Acceso el 28 de marzo de 2005.

19. Organización de Naciones Unidas. Objetivos de desarrollo de la ONU para el milenio. Nueva York: ONU; 2000. Hallado en: http:/ /www.un.org/spanish/ millen niumgoals/. Acceso el 7 de abril de 2005.

20. Pan American Health Organization. Health in the Americas, 2002. Vol. 1. Washington, D.C.: PAHO; 2002.

21. Inter-American Development Bank. Facing up to inequality in Latin America. Washington, D.C.: IDB; 1998. Hallado en: http://www.iadb.org/exr/english/ PRESS_PUBS/ipintr.htm. Acceso el 7 de abril de 2005.

22. Pan American Health Organization. Equity and health: views from the Pan American Sanitary Bureau. Washington, D.C.: PAHO; 2001.

23. Torres C, Mújica OJ. Salud, equidad y los objetivos de desarrollo del milenio. Rev Panam Salud Publica. 2004;15(6): 430-9.

24. Health-related millennium development goals out of reach for many countries. [WHO news]. Bull World Health Organ. 2004;82(2):156-7.

25. Haines A, Cassels A. Can the millennium development goals be attained? Br Med J. 2004;329:394-7.

26. Labonte R, Schrecker T, Gupta AS. A global health equity agenda for the G8 summit. Br Med J. 2005;330:533-6.

27. San Sebastian M, Hurtig AK. From NAFTA to FTAA? The impact of deregulation of trade on social and health conditions of people of the Americas. Pan Am J Public Health. 2004;16(4):272-8. 